\title{
All Is Not Lost: Positive Behaviors in Alzheimer's Disease and Behavioral-Variant Frontotemporal Dementia with Disease Severity
}

\author{
Akira Midorikawa $a^{\mathrm{a}, \mathrm{b}, *}$, Cristian E. Leyton ${ }^{\mathrm{b}, \mathrm{c}, \mathrm{d}}$, David Foxe $^{\mathrm{b}, \mathrm{d}}$, Ramon Landin-Romero $^{\mathrm{b}, \mathrm{d}, \mathrm{e}}$, \\ John R. Hodges ${ }^{\mathrm{b}, \mathrm{d}, \mathrm{e}}$ and Olivier Piguet $\mathrm{t}^{\mathrm{b}, \mathrm{d}, \mathrm{e}, *}$ \\ ${ }^{a}$ Department of Psychology, Faculty of Letters, Chuo University, Tokyo, Japan \\ ${ }^{\mathrm{b}}$ Neuroscience Research Australia, Sydney, Australia \\ ${ }^{\mathrm{c}}$ Faculty of Health Sciences, The University of Sydney, Lidcombe, NSW, Australia \\ ${ }^{\mathrm{d}}$ Australia Research Council Centre of Excellence in Cognition and its Disorders, Sydney, Australia \\ ${ }^{\mathrm{e}}$ School of Medical Sciences, The University of New South Wales, Sydney, Australia
}

Accepted 9 June 2016

\begin{abstract}
. of these changes has not been formally investigated. bvFTD patients, regardless of disease severity. questionnaire development

*Correspondence to: Dr Akira Midorikawa, Department of Psychology, Faculty of Letters, Chuo University, 742-1 Higashinakano, Hachioji, 192-0393 Tokyo, Japan. Tel.: +81 0426743742 ; E-mail: green@tamacc.chuo-u.ac.jp and Olivier Piguet, Neuroscience Research Australia, Barker St, Randwick, NSW 2031, Australia. E-mail: o.piguet@neura.edu.au.
\end{abstract}

Background: Anecdotal evidence indicates that some patients with dementia exhibit novel or increased positive behaviors, such as painting or singing, after the disease onset. Due to the lack of objective measures, however, the frequency and nature

Objective: This study aimed to systematically identify changes in these behaviors in the two most common younger-onset dementia syndromes: Alzheimer's disease (AD) and behavioral-variant frontotemporal dementia (bvFTD).

Methods: Sixty-three caregivers of patients with dementia (32 caregivers of AD patients and 31 caregivers of bvFTD patients) participated in the study. Caregivers rated the presence and frequency of positive and negative behavior changes after the onset of dementia using the Hypersensory and Social/Emotional Scale (HSS) questionnaire, focusing on three domains: sensory processing, cognitive skills, and social/emotional processing. Six composites scores were obtained reflecting these three domains (two composite scores for each domain). Differences across scores and ratios of increased and decreased behaviors were analyzed between AD and bvFTD, at different disease severity levels.

Results: After disease onset, significant changes in the sensory processing domain were observed across disease severity levels, particularly in AD. Composite scores of the other domains did not change significantly. Importantly, however, some novel or increased positive behaviors were present in between 10\% (Music activities) and 70\% (Hypersensitivity) of AD and

Conclusions: We provide the first systematic investigation of positive behaviors in AD and bvFTD. The newly developed HSS questionnaire is a valid measure to characterize changes and progression of positive behaviors in patients with dementia.

Keywords: Alzheimer's disease, behavioral symptoms, caregivers, factor analysis, frontotemporal dementia, progression,

\section{INTRODUCTION}

Dementia is associated with widespread changes in cognition and social interactions [1], as well as with the emergence of abnormal behavior and psychiatric symptomatology [2]. These changes tend to 
become more pronounced with disease progression. Anecdotal evidence, however, indicates that creative "positive" skills can emerge or improve in patients with dementia, such as painting, drawing, or singing skills -which were not necessarily part of the individual's repertoire prior to disease onset. For example, novel drawing skills have been reported after the onset of frontotemporal dementia (FTD) [3-7], in Alzheimer's disease (AD) [8], and in Parkinson's disease [9]. Other novel activities, such as doing puzzles [10], listening to music [11], or playing an instrument [12] have also been reported in dementia patients as the disease progresses. These reports indicate that individuals with dementia have the potential for exhibiting novel, not previously seen, activities, against a background of cognitive and functional decline.

In addition to these reported changes in cognitive skills, changes in sensory functions with dementia have also been reported, such as an increase of sensitivity or hypersensitivity for pain or temperature [13, 14]. These findings suggest that somatosensory functions are abnormal in some patients with dementia. Whether other senses are also affected in dementia remains unknown, although increased sensitivity to intense light and loud sound stimuli is commonly reported following traumatic brain injury [15]. These findings would suggest that diffuse brain damage is likely to interfere with inhibitory processes and the capacity of the brain to modulate and control the flow of sensory information.

Changes in social/emotional processing have been widely documented in dementia [16]. Emergence of "negative" behavioral changes, such as apathy, anxiety, and depression have been commonly described in mild cognitive impairment (MCI) [17] and AD patients [18]. Importantly, heightened emotional empathy has also been reported in these clinical groups compared to controls [19], a change not explained by a generalized mood disorder, but rather a specific change in interpersonal emotional reactivity [19].

In summary, existing evidence points towards the emergence or increase in positive behaviors in some dementia patients with disease progression. Such behaviors have been reported in different domains, including cognition, processing of sensory and social/emotional information. These changes, however, have not been systematically investigated and their examination is rarely part of the clinical investigation of dementia patients. Indeed, the characteristics, severity, and prevalence of these features across dementia syndromes are currently unknown.

This study aimed to systematically determine the presence and characteristics of positive behavioral changes across disease severity in the two dementia syndromes where these changes have been most commonly reported: AD and bvFTD. The presence and frequency of positive behaviors were established using the Hypersensory and Social/Emotional Scale (HSS), a newly developed caregiver questionnaire. Caregiver-based questionnaires are a particularly suitable measure of behavior of patients in the advanced stages of dementia and/or where formal cognitive testing is no longer suitable or appropriate.

\section{MATERIALS AND METHODS}

\section{Development of the Hypersensory \\ and Social/Emotional Scale (HSS)}

\section{Operationalization and item selection of the HSS}

Question items from the HSS were derived from previous reports of novel or increased positive behaviors in dementia and focused on three domains: sensory processing, cognitive skills, and social/emotional processing. Items from the HSS investigating behaviors related to the sensory processing domain were selected from previously developed hypersensitivity questionnaires for autism spectrum disorder [20, 21]. Items measuring behaviors related to the other two domains were selected by consensus after discussion among clinicians. From a pool of 48 items, 19 were included in the HSS as they were thought to unequivocally characterize positive changes in the three domains of interest (see Supplementary Table 1 for details of the HSS). For each item, behavior frequency was rated using a four point-scale from 0 to 3 ( 0 Never, 1 Rarely, 2 Sometimes, 3 Frequently; see Supplementary Table 1). To detect differences between premorbid and current state, caregivers were asked to rate the frequency of each behavior in the patients at two different time points, namely "Before the illness" and "At the present time".

\section{Study participants for the development \\ of the questionnaire}

The internal structure of the HSS was tested using participants from FRONTIER, the frontotemporal dementia research clinic in Sydney, Australia. Since 2007 , FRONTIER has recruited a large $(>500)$ cohort 
Table 1

Clinical and demographic characteristics of study participants

\begin{tabular}{lccc}
\hline & $\mathrm{AD}(n=32)$ & bvFTD $(n=31)$ & $p$ value \\
\hline Age (years) & $67.1(8.8)$ & $63.8(8.7)$ & $\mathrm{ns}^{\mathrm{a}}$ \\
Sex (Male/Female) & $18 / 14$ & $18 / 13$ & $\mathrm{~ns}^{\mathrm{b}}$ \\
Disease duration (y) & $6.4(4.3)$ & $4.4(2.7)$ & $0.03^{*, \mathrm{a}}$ \\
CDR $(0.5 / 1 / 2)$ & $8 / 17 / 7$ & $7 / 17 / 7$ & $\mathrm{~ns}^{\mathrm{b}}$ \\
\hline
\end{tabular}

Numerical values are illustrated in mean (SD). AD, Alzheimer's disease; bvFTD, behavioral-variant frontotemporal dementia; CDR, global clinical dementia rating scale; $\mathrm{ns}=p>0.05$. ${ }^{\mathrm{a}}$ Student $t$-test analysis; ${ }^{b} \mathrm{Chi}$-square analysis, ${ }^{*}$ denotes significant differences between AD and bvFTD.

of patients with frontotemporal dementia (FTD), FTD-related conditions, AD, and other youngeronset dementias. For this study, we approached all relevant caregivers of patients who had been involved in our research program since its inception in 2007. In order to characterize novel behaviors in a broad spectrum of syndromes and diagnoses, the selection criteria included different subtypes of dementia such as FTD variants (behavioral-variant of FTD: bvFTD, semantic dementia, progressive non-fluent aphasia), $A D$ variants (typical $A D$ : $A D$, logopenic progressive aphasia, and posterior cortical atrophy), and corticobasal syndrome. Details of the demographic and clinical characteristics of the study participants are presented in Supplementary Table 2.

Two-hundred-and-sixty-one caregivers of patients with dementia were contacted by mail and sent the HSS questionnaire as well as the frontotemporal lobar dementia-modified Clinical Dementia Rating (FTLD-modified CDR) [22], in order to establish disease severity, of whom 160 returned the questionnaire (61.3\%). An additional 33 caregivers were approached to participate as part of their scheduled visit to our research program. Eight questionnaires were from deceased patients and had to be excluded and a further eight questionnaires were excluded due to missing data for $>20 \%$ of the items. Thus, a total of 185 valid questionnaires (152 from the mailing sample and 33 collected directly) were included. A detailed flow chart of the data collection procedure is shown Supplementary Fig. 1.

Ethics approval was given by the South Eastern Sydney Local Health District and the University of New South Wales.

\section{Statistical analysis for item selection}

Statistical analyses were performed using SPSS 22.0 (IBM Corp., Armonk, NY) and R [23]. For each item, a subtraction score ("At the present time" "Before the illness") was calculated which ranged between -3 and +3 . A positive score indicated the emergence or an increase in behavior after the onset of the disease, whereas a negative score reflected a decrease in behavior after disease onset and a score of 0 for no change. The total missing value was small (1.38\%; ranging between $0-5.1 \%$ for each item), 126 out of 177 participants (71.2\%) had completed all items. As such, the Expectation Maximization imputation method was used to impute missing values.

Exploratory factor analysis using the maximum likelihood method with promax rotations was carried out to determine the underlying factor structure of the HSS (Supplementary Table 3). The number of factors to retain was established using parallel analyses $[24,25]$. Three factors were identified, accounting for $34.2 \%$ of the score variance and aligned with our three domains of interest. The first factor, labeled Social Factor, contained 6 items, the second factor labeled Sensory Factor, comprised 7 items, and the final factor, labeled Cognitive Factor, contained 6 items. One item from this factor was removed due to low factor loadings (less than 0.35) (Supplementary Table 3). Clinically, however, the underlying structure of each factor was not uniform. As such, each factor was subjected to additional exploratory factor analysis using parallel analysis and the maximum likelihood method with promax rotations. These analyses uncovered two distinct components to each factor. The complete description of the items associated with each component and its factor is provided in Table 2.

\section{Emergence of positive behaviors in $A D$ and bvFTD}

\section{Participants}

From the overall cohort whose data were included the factor analysis, caregivers of patients diagnosed with $\mathrm{AD}(n=31)$ or with probable bvFTD $(n=32)$ participated in this study. Study participants were recruited from FRONTIER, the frontotemporal dementia research clinic based at Neuroscience Research Australia (NeuRA) in Sydney. Patients were seen by an experienced neurologist and a diagnosis was made in line with current diagnostic criteria for $\mathrm{AD}$ [26] and bvFTD [27] according to the neurological assessment, comprehensive neuropsychological assessment and structural brain 3T magnetic resonance imaging (MRI). All patients scored between 0.5 and 2 on the clinical dementia rating (CDR) [28], derived from the caregiver self-completed questionnaire version of the 
Table 2

Separate exploratory factor analysis on each factor

\begin{tabular}{|c|c|c|}
\hline Sensory Factor & $\mathrm{C} 1$ & $\mathrm{C} 2$ \\
\hline \multicolumn{3}{|l|}{ Component 1: Sensitivity to details $(\mathrm{Cl})$} \\
\hline 19. Is rigid and particular regarding the way items must be organized or ordered (for example documents on a desk) & 0.88 & -0.14 \\
\hline 8. Is sensitive to objects that are misaligned (for example a painting that is not straight on the wall) & 0.52 & 0.12 \\
\hline 1. Is sensitive to details (for example, tiny specks of dust on the table or rubbish on the road) & 0.47 & 0.18 \\
\hline \multicolumn{3}{|l|}{ Component 2: Hypersensitivity (C2) } \\
\hline 6. Dislikes loud noises (responds negatively to unexpected loud noises) & -0.06 & 0.60 \\
\hline 5. Hates the feel or texture of certain foods in the mouth & -0.03 & 0.52 \\
\hline 7. Complains about bright lights & 0.08 & 0.52 \\
\hline 12. Draws the curtains in late afternoon even when outside is still bright & 0.12 & 0.46 \\
\hline Cognitive Factor & $\mathrm{C} 1$ & $\mathrm{C} 2$ \\
\hline \multicolumn{3}{|l|}{ Component 1: Language related activities (C1) } \\
\hline 3. Reminisces mostly about recent events (recent weeks) & 1.00 & -0.05 \\
\hline 2. Likes to write everything down & 0.31 & 0.30 \\
\hline \multicolumn{3}{|l|}{ Component 2: Visuospatial related activities (C2) } \\
\hline 11. Likes taking photographs & -0.06 & 0.67 \\
\hline 10. Likes drawing pictures & 0.01 & 0.51 \\
\hline 18. Likes doing puzzles & 0.16 & 0.43 \\
\hline Social Factor & $\mathrm{C} 1$ & $\mathrm{C} 2$ \\
\hline \multicolumn{3}{|l|}{ Component 1: Social attitude (C1) } \\
\hline 13. Tries to help out a person in need (for example, will comfort someone who is hurt or sad) & 0.90 & -0.16 \\
\hline 16. Shows affection towards family members or friends & 0.51 & 0.24 \\
\hline 14. Makes eye contact easily with other people & 0.50 & 0.15 \\
\hline 4. Has a positive reaction to babies (smiles and talks to the baby) & 0.37 & 0.26 \\
\hline \multicolumn{3}{|l|}{ Component 2: Music activities (C2) } \\
\hline 9. Listens to music & -0.03 & 0.69 \\
\hline 15. Sings along to music on CDs, radio, or television & 0.02 & 0.59 \\
\hline
\end{tabular}

Bold font indicates a loading greater than 0.30 .

Table 3

Emerging behaviors in Alzheimer's disease and behavioral-variant frontotemporal dementia (Mean $\pm S D$ )

\begin{tabular}{|c|c|c|c|c|c|c|c|c|c|}
\hline & \multirow[t]{2}{*}{$\mathrm{AD}(n=32)$} & \multirow[t]{2}{*}{$\operatorname{bvFTD}(n=31)$} & \multicolumn{2}{|c|}{ Diagnosis } & \multicolumn{2}{|c|}{ CDR } & \multicolumn{2}{|c|}{ Interaction } & \multirow{2}{*}{$\begin{array}{c}\text { Post hoc } \\
\text { CDR }\end{array}$} \\
\hline & & & $F$ & $p$ & $F$ & $p$ & $F$ & $p$ & \\
\hline \multicolumn{10}{|l|}{ Sensory Factor } \\
\hline Hypersensitivity & $0.51 \pm 0.48$ & $0.50 \pm 0.58$ & 0.04 & ns & 1.94 & $\mathrm{~ns}$ & 0.39 & ns & - \\
\hline Sensitivity to detail & $0.32 \pm 0.98$ & $0.01 \pm 0.92$ & 0.25 & ns & 0.92 & ns & 1.81 & ns & - \\
\hline \multicolumn{10}{|l|}{ Cognitive Factor } \\
\hline Language related activities & $-0.09 \pm 1.06$ & $-0.43 \pm 1.24$ & 0.30 & ns & 5.70 & $* *$ & 1.02 & ns & $0.5>1^{*} ; 0.5>2^{* *}$ \\
\hline Visuospatial related activities & $-0.29 \pm 0.86$ & $-0.16 \pm 0.82$ & 0.56 & ns & 3.10 & $\dagger$ & 0.60 & ns & - \\
\hline \multicolumn{10}{|c|}{ Social Factor } \\
\hline Music activities & $-0.09 \pm 0.72$ & $-0.39 \pm 1.06$ & 1.41 & ns & 0.34 & $\mathrm{~ns}$ & 0.14 & ns & - \\
\hline Social attitude & $-0.09 \pm 0.62$ & $-0.60 \pm 0.99$ & 2.24 & ns & 1.10 & $\mathrm{~ns}$ & 1.83 & ns & - \\
\hline
\end{tabular}

$\mathrm{AD}$, Alzheimer's disease; bvFTD, behavioral-variant frontotemporal dementia; CDR, global clinical dementia rating scale; ns $=p>0.05$. ${ }^{*} p<0.05 ;{ }^{* *} p<0.01 ;{ }^{\dagger}$ denotes trend towards significance in observed differences $(p<0.10)$.

FTLD-modified CDR scale [22]. The HSS questionnaire and FTLD-modified CDR were mailed to the caregivers or given directly to caregivers when they visited the institute during the patient's routine annual clinical assessment.

\section{Statistical analyses}

Statistical analyses were performed using SPSS 22.0 (Armonk, NY: IBM Corp). Demographic data were compared using independent $t$-tests for parametric data and chi-square test for categorical data. For the HSS, six composite scores were derived
(1 for each component associated with the three factors), by averaging the scores of the questions within each component. For each composite score, the magnitude of change from 0 (reflecting no change in behavior between before disease onset and current situation) was investigated using single-sample $t$-tests. In addition, each composite score was subjected to a two-way (diagnosis $x$ severity) analysis of variance (ANOVA) followed by post hoc pairwise comparisons with Bonferroni correction. All statistical tests were two-sided and statistical significance was set at the $p$ value of $<0.05$. 


\section{RESULTS}

Demographic and clinical characteristics of the $A D$ and bvFTD samples

The clinical and demographic characteristics for the two dementia groups are shown in Table 1. The clinical groups did not significantly differ with respect to age, sex distribution, and disease severity; however, disease duration was significantly shorter in bvFTD than in AD.

\section{Magnitude of positive behavior changes in $A D$ and $b v F T D$}

Composite scores of the factors reflecting the three behavior domains are shown in Table 3. Single sample $t$-tests were carried out to characterize changes with disease severity for each composite score in each group (Fig. 1). The AD group showed a significant increase in the Sensory Factor for Hypersensitivity (CDR 0.5, 1, and 2) and Sensitivity to detail (CDR 1) and a marginal increase at CDR 0.5 for Language related activities. A decrease in behavior was also observed in $\mathrm{AD}$, particularly in the severe stages of the disease. Specifically, the AD group showed a significant decrease in Language related activities at CDR 2, when compared to 0 and 0.5 global CDR scores. Decrease from 0 in Visuospatial activities was approaching significance at the CDR 1 and CDR 2 scores. In the bvFTD, a significant increase in Hypersensitivity was observed at CDR 1 and CDR 2 and a significant decrease in Social attitude at CDR 1. No other significant changes were observed on the remaining composite scores in this group.
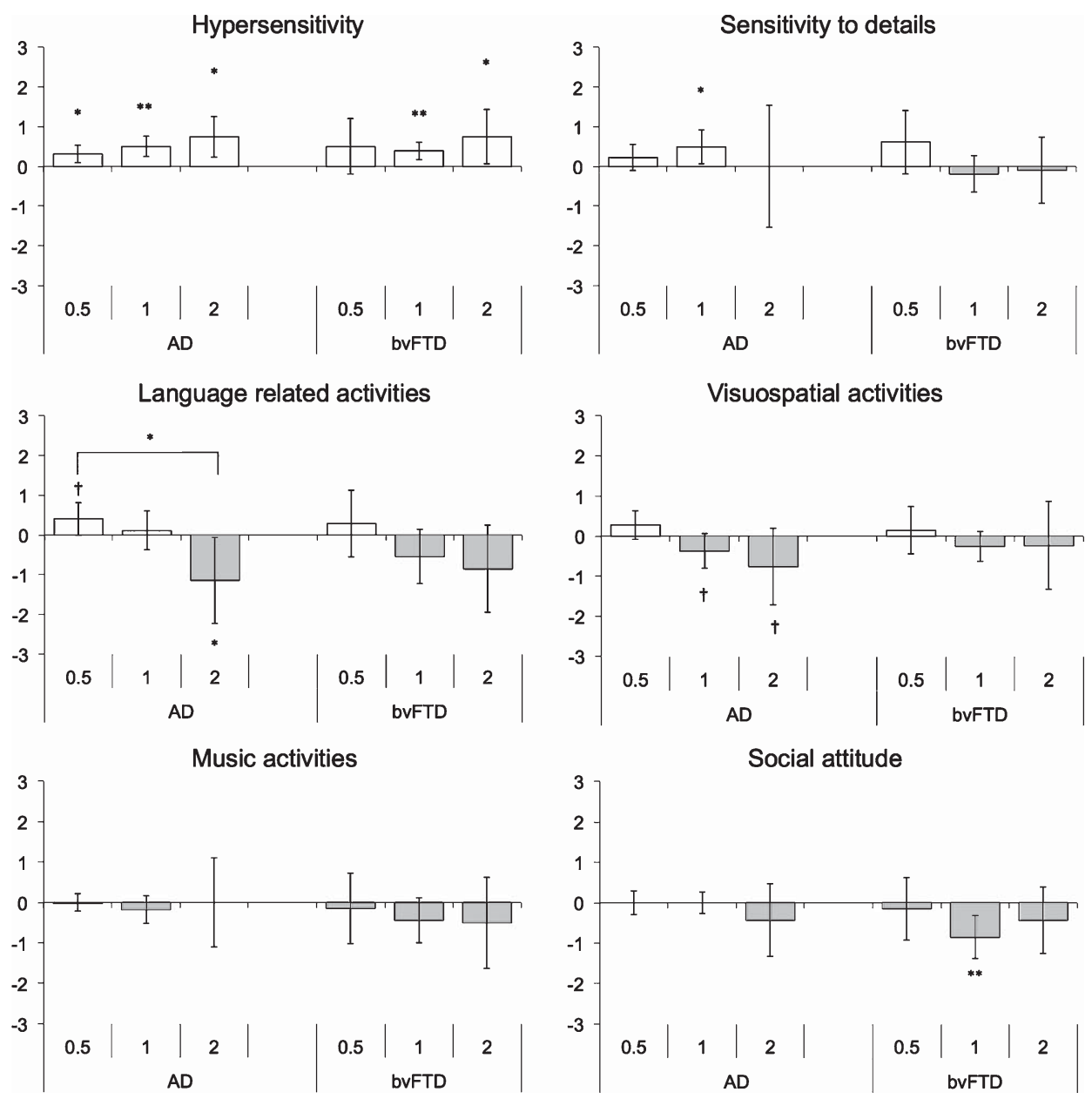

Fig. 1. Mean component scores across disease severity in Alzheimer's disease and behavioral-variant frontotemporal dementia. Error bars indicate $\pm 95 \%$ CI for each disease severity (CDR 0.5/1/2). White bars denote increase in behavior and gray bars denote decrease in behavior. ${ }^{*} p<0.05 ;{ }^{* *} p<0.01{ }^{\dagger}$ denotes trend toward significance in observed differences $(p<0.10)$. 
These changes in behavior are further illustrated by depicting the proportion of patients exhibiting changes in composite scores for each factor in both clinical groups (Fig. 2). This figure shows that the majority of $\mathrm{AD}$ and bvFTD patients experienced increased hypersensitivity at all stages of the disease. While increased sensitivity to details was also common in both groups, a growing proportion of $\mathrm{AD}$ and bvFTD patients showed a decrease as the disease severity worsens. Over $60 \%$ of AD patients showed an increase in language-related activities in the early stage of the disease, with none experiencing a decrease. With worsening disease severity, however, language-related activities declined. This was most pronounced at CDR 2 where over 75\% of bvFTD and $\mathrm{AD}$ patients showed a decline in these activities. In bvFTD, although an increase in language-related activities was also present early on, a reduction in these activities was observed in a small proportion of patients as well. This proportion became much larger with disease progression, affecting up to $80 \%$ of patients at CDR 2.

The proportion of increased visuospatial activities was small in both AD and bvFTD. Indeed, the largest proportion of $\mathrm{AD}$ and bvFTD patients showed no change in their engagement in these activities, although a pronounced decrease became apparent with disease progression. Around 10-20\% of patients exhibited a positive change in music activities, a proportion which remained stable with increasing disease severity. Nevertheless, the proportion of patients showing a decline in this aptitude became larger as the disease worsened. A similar pattern was observed with regard to social attitude, with the decline on this score being, not surprisingly, particularly pronounced in bvFTD.

In summary, this figure demonstrates that novel or increased behavior are present in both $\mathrm{AD}$ and bvFTD and are not limited to a particular domain. Importantly, previously reported emergence in music and visuospatial activities appear to be rare and are not limited to bvFTD. In addition, when present, these are found at all disease severity levels.

\section{Comparing positive behavior changes between $A D$ and $b v F T D$}

Two-way ANOVAs were carried out separately on all composite scores (Table 3). These analyses showed no significant main effect of diagnosis or interaction effects. A significant main effect of disease severity was observed for the composite score of Language related activities $(F(2,57)=5.70$, $p=0.01$ ), with post hoc tests indicating significantly higher scores at the CDR 0.5 than at the CDR 2 and CDR 1 levels. No other composite score showed a significant change with disease severity, although a trend was present for the composite score of Visuospatial related activities $(F(2,57)=3.10, p=0.05)$.

\section{DISCUSSION}

This study examined systematically the presence of novel or increased positive behaviors in $\mathrm{AD}$ and bvFTD at different disease stages across three domains (sensory processes, cognitive skills, and social/emotional processing) using the HSS, a newly developed caregiver-based questionnaire.

Overall, AD patients displayed an increase in positive behaviors in both components of the Sensory Factor after disease onset (i.e., Hypersensitivity and Sensitivity to details). In the bvFTD group, however, an increase in that factor was limited to Hypersensitivity and was found only at the severe disease stages (CDR 1 and CDR 2). When present, changes in the remaining scores were characterized by a decline, rather than an increase, in positive behaviors with increasing disease severity (AD: Language related activities; bvFTD: Social attitude). Importantly, examination of the differences between $\mathrm{AD}$ and bvFTD on these composite scores, and of the effect of disease severity, demonstrated that the two clinical groups did not differ on any of these measures. An effect of disease severity was found for only one composite score. The Language related activities score, which reflects the Cognitive Factor, was found to decline with increasing disease severity (CDR 0.5 > CDR 2).

Unlike previous research investigating sensory processing in dementia, which mostly focused on somatosensory functions [13, 14, 29], the HSS Sensory Factor measures the emergence of behaviors related to various sensory functions, including sensitivity to brightness, loudness, food texture, or the attention to detailed information. We found positive changes affecting the Sensory Factor in AD, especially with the component Hypersensitivity, which were present across the spectrum of disease severity. Reduced tolerance to sound or light has previously been shown to correlate with both cognitive and emotional disturbances following traumatic brain injury [15]. In addition, hypersensitivity is commonly observed in autism spectrum disorders [20,21] with 

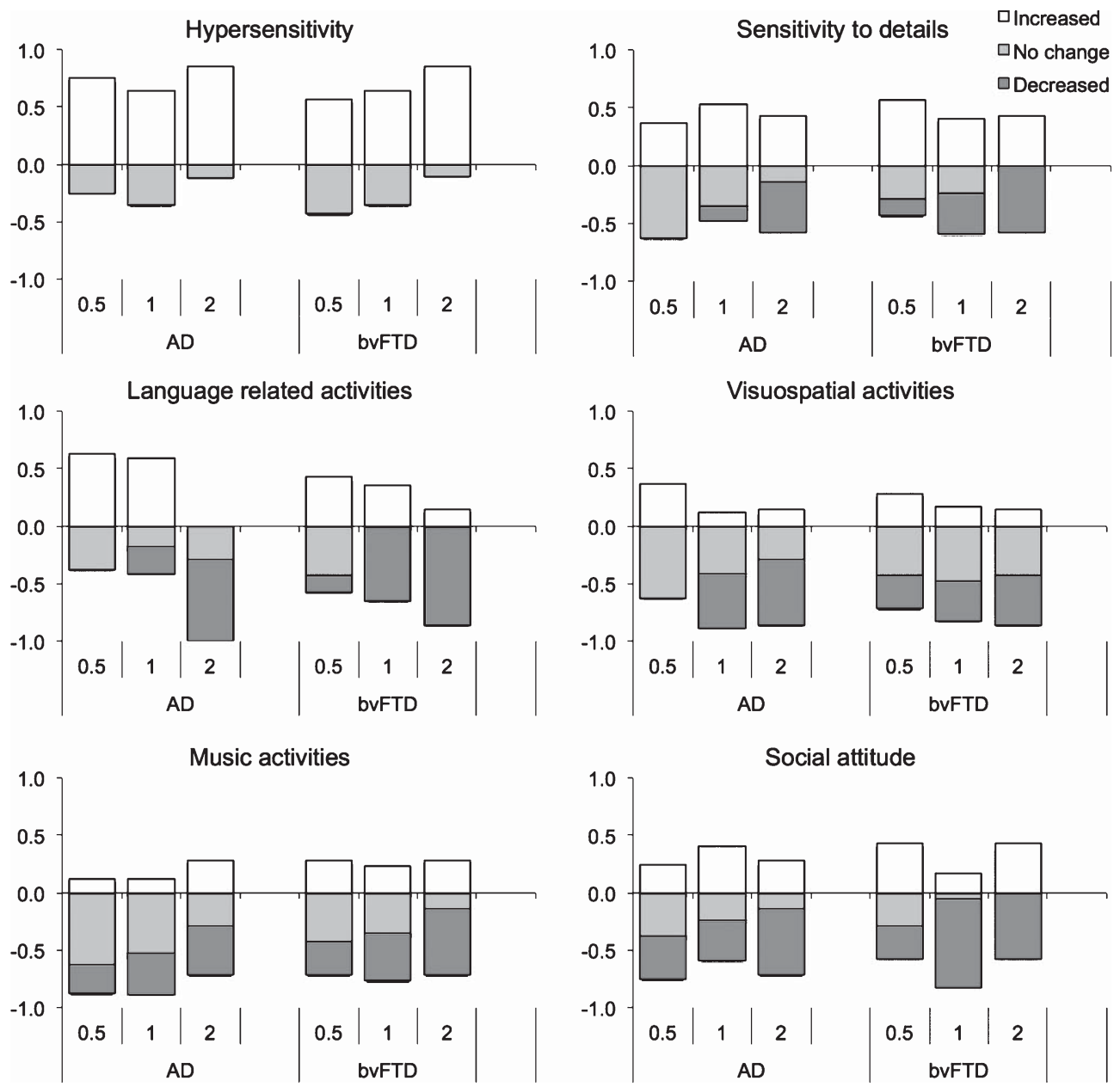

Fig. 2. Proportion of patients exhibiting increased, no change, and decreased behaviors at different levels of disease severity in Alzheimer's disease and behavioral-variant frontotemporal dementia.

sensory stimulation being one of the main causes of panic or discomfort in these patients [20]. Our findings indicate that hypersensitivity to sensory stimuli appears to be a general phenomenon in patients with brain disorders, and not specific to traumatic brain injury or autism spectrum disorders patients. As such, an increase in the Sensory Factor may be related to the presence of negative behaviors or behavioral and psychological symptoms of dementia.

In the Cognitive Factor, novel or increased behaviors were not limited to bvFTD, but were also observed in $\mathrm{AD}$, particularly in the early stages of the disease (CDR 0.5). Unlike in bvFTD, these skills tended to dissipate with disease severity. On the other hand, and while categorized with Social Factor, the component Music activities was stable. This finding accords well with the fact that music activities appear to rely on widespread brain networks that are different from other cognitive activities. The component Social attitude includes items related to family affection and positive reactions to babies. While increased empathy and emotional contagion have been reported in MCI and AD [19], bvFTD is generally reported to be accompanied by negative behaviors, such as social inappropriateness [30] and reduction in empathy and emotion recognition [31-33]. Here, the overall changes were not as pronounced as would have been anticipated in the bvFTD group. Indeed, a reported decline in social attitude did not differ from 0 in this group, except at the CDR 1 level. Importantly, a subset of AD and bvFTD patients exhibited increased social behaviors at all levels of disease severity. This finding indicates that positive prosocial behaviors are present in some bvFTD patients, which has implications for the management of these patients. Whether these individuals differ 
from the broad bvFTD population on other aspects of prosocial behaviors remains unknown. The smaller change in the AD group observed in this factor compared with previous studies may reflect differences in the instruments used: "self"-oriented behaviors [19, 34] versus "other"-oriented behaviors here.

The biological mechanisms underlying the emergence of these positive behaviors remain unclear. One explanation is that the progressive pathological processes, which start relatively focally, will result in the release of 'spared' functions, mediated by brain regions not (or less) affected by the disease [3]. As such, in bvFTD patients, emergence of artistic abilities would arise because of the combination of anterior brain region pathology with comparatively spared posterioparietal regions, particularly in the right (non-dominant) hemisphere [35]. A similar mechanism has been proposed supporting the emergence of artistic abilities following anterior temporal lobe resection for the treatment of intractable epilepsy [36]. Importantly, however, although predominantly reported in FTD patients, our study revealed that emergence of positive behaviors is also found in $\mathrm{AD}$, a disease with marked pathological changes involving the parietal and temporal lobe brain regions. In AD patients, previously reported changes in artistic abilities were said to take the form of stylistic change, from realist to freeform but with preserved artistic qualities [35].

Noteworthy is that in both groups increase in behavior was most marked in the sensory components (Hypersensitivity, Sensitivity to details). Disrupted processing of temperature and pain signals have been recently reported in bvFTD and AD [14], associated with atrophy of the anterior insula, particularly on the right. Nevertheless, within each dementia type, the proportion of individuals exhibiting these positive behavioral changes remains relatively small, which indicate that further investigations will be needed to determine their specific biological mechanisms.

To our knowledge, this study is the first to systematically explore the emergence or increase of positive behaviors in AD and bvFTD at different levels of disease severity. The caregiver-based approach allowed us to determine the type and frequency of these behaviors, particularly in the later stages of the disease when a formal clinical or neuropsychological examination is no longer possible. Our study has some limitations, which are intrinsic to caregiver-based questionnaires. Often caregivers of individuals with dementia experience difficulty in establishing behavioral changes after the onset of the disease due to their insidious and progressive course. As such, the severity and frequency of change may be under-reported. Our analyses, however, based on difference scores between "Before the illness" and "At the present time" yielded an appropriate distribution of changes across disease severity stages. This indicates that caregivers were able to determine the onset of the behaviors of interest. Secondly, based on factor analysis, Music related activities were grouped as part of the Social rather than the Cognitive Factor, with robust findings. While surprising at first glance, music and singing activities have strong social functions and help with interacting with others $[37,38]$. Therefore, compared to other cognitive activities, music related activities were more aligned with social settings. Finally, our three domains may appear reductionist and may fail to capture specific changes in behavior. This approach, however, represents an important first step toward an understanding of the complexity of behavioral changes that occur as the dementia unfolds. As such, information arising from this investigation will facilitate and promote the caregiver awareness of the patient's positive attitudes.

A better understanding of the emergence of novel abilities may provide an additional window for targeted and individualized patient-centered interventions and will help with the management of patients with dementia, particularly in the later stages of the disease. Indeed, a shift in the conceptualization of dementia emphasizing preserved or increased abilities, while not negating the presence of marked functional and cognitive loss, has been shown to bring benefits not only to caregivers but also to the patients themselves [39]. Finally, the systematic characterization of emergent positive behaviors will contribute to improving our understanding of these dementia syndromes.

\section{ACKNOWLEDGMENTS}

The authors are grateful to the participants and their families for supporting this research.

This work was supported in part by funding to ForeFront, a collaborative research group dedicated to the study of frontotemporal dementia and motor neuron disease, from the National Health and Medical Research Council (NHMRC) (APP1037746) and the Australia Research Council (ARC) Centre of Excellence in Cognition and its Disorders Memory Node (CE11000102). AM is supported by Chuo University Overseas Research Program, and Japan 
Society for the Promotion of Science (KAKENHI 15K04191). CL is supported by a NHMRC - ARC Dementia Research Development Fellowship (APP 1102969). RLR is supported by the ARC Centre of Excellence in Cognition and its Disorders Memory Node (CE11000102). OP is supported by an NHMRC Senior Research Fellowship (APP1103258).

Authors' disclosures available online (http://j-alz. com/manuscript-disclosures/16-0440r1).

\section{SUPPLEMENTARY MATERIAL}

The supplementary material is available in the electronic version of this article: http://dx.doi. org/10.3233/JAD-160440.

\section{REFERENCES}

[1] Weintraub S, Wicklund AH, Salmon DP (2012) The neuropsychological profile of Alzheimer disease. Cold Spring Harb Perspect Med 2, a006171.

[2] Lyketsos CG, Lopez O, Jones B, Fitzpatrick AL, Breitner J, DeKosky S (2002) Prevalence of neuropsychiatric symptoms in dementia and mild cognitive impairment: Results from the cardiovascular health study. JAMA 288, 14751483.

[3] Miller BL, Cummings J, Mishkin F, Boone K, Prince F, Ponton M, Cotman C (1998) Emergence of artistic talent in frontotemporal dementia. Neurology 51, 978-982.

[4] Mendez MF (2004) Dementia as a window to the neurology of art. Med Hypotheses 63, 1-7.

[5] Midorikawa A, Fukutake T, Kawamura M (2008) Dementia and painting in patients from different cultural backgrounds. Eur Neurol 60, 224-229.

[6] Mell JC, Howard SM, Miller BL (2003) Art and the brain: The influence of frontotemporal dementia on an accomplished artist. Neurology 60, 1707-1710.

[7] Drago V, Foster PS, Trifiletti D, FitzGerald DB, Kluger BM, Crucian GP, Heilman KM (2006) What's inside the art? The influence of frontotemporal dementia in art production. Neurology 67, 1285-1287.

[8] Chakravarty A (2011) De novo development of artistic creativity in Alzheimer's disease. Ann Indian Acad Neurol 14, 291.

[9] Walker RH, Warwick R, Cercy SP (2006) Augmentation of artistic productivity in Parkinson's disease. Mov Disord 21, 285-286.

[10] Green HAC, Patterson K (2009) Jigsaws-a preserved ability in semantic dementia. Neuropsychologia 47, 569-576.

[11] Fletcher PD, Downey LE, Witoonpanich P, Warren JD (2013) The brain basis of musicophilia: Evidence from frontotemporal lobar degeneration. Front Psychol 4, 347.

[12] Cho H, Chin J, Suh MK, Kim HJ, Kim YJ, Ye BS, Lee NK, Kim EJ, Seo SW, Na DL (2015) Postmorbid learning of saxophone playing in a patient with frontotemporal dementia. Neurocase 21, 767-772.

[13] Snowden JS, Bathgate D, Varma A, Blackshaw A, Gibbons ZC, Neary D (2001) Distinct behavioural profiles in frontotemporal dementia and semantic dementia. J Neurol Neurosurg Psychiatry 70, 323-332.
[14] Fletcher PD, Downey LE, Golden HL, Clark CN, Slattery CF, Paterson RW, Rohrer JD, Schott JM, Rossor MN, Warren JD (2015) Pain and temperature processing in dementia: A clinical and neuroanatomical analysis. Brain 138, 33603372.

[15] Bohnen N, Twijnstra A, Wijnen G, Jolles J (1992) Recovery from visual and acoustic hyperaesthesia after mild head injury in relation to patterns of behavioural dysfunction. $J$ Neurol Neurosurg Psychiatry 55, 222-224.

[16] Kumfor F, Piguet O (2012) Disturbance of emotion processing in frontotemporal dementia: A synthesis of cognitive and neuroimaging findings. Neuropsychol Rev 22, 280-297.

[17] Geda YE, Roberts RO, Knopman DS, Petersen RC, Christianson TJH, Pankratz VS, Smith GE, Boeve BF, Ivnik RJ, Tangalos EG, Rocca WA (2008) Prevalence of neuropsychiatric symptoms in mild cognitive impairment and normal cognitive aging. Arch Gen Psychiatry 65, 1193.

[18] Spalletta G, Musicco M, Padovani A, Perri R, Fadda L, Canonico V, Trequattrini A, Pettenati C, Caltagirone C, Palmer K, Rozzini L (2010) Neuropsychiatric symptoms and syndromes in a large cohort of newly diagnosed, untreated patients with Alzheimer disease. Am J Geriatr Psychiatry 18, 1026-1035.

[19] Sturm VE, Yokoyama JS, Seeley WW, Kramer JH, Miller BL, Rankin KP (2013) Heightened emotional contagion in mild cognitive impairment and Alzheimer's disease is associated with temporal lobe degeneration. Proc Natl Acad Sci U S A 110, 9944-9949.

[20] Robertson AE (2012) Sensory experiences of individuals with Autism Spectrum Disorder and autistic traits: A mixed methods approach. PhD Thesis, University of Glasgow, http://theses.gla.ac.uk/3769/

[21] Tomchek SD, Dunn W (2007) Sensory processing in children with and without autism: A comparative study using the short sensory profile. Am J Occup Ther 61, 190-200.

[22] Knopman DS, Kramer JH, Boeve BF, Caselli RJ, GraffRadford NR, Mendez MF, Miller BL, Mercaldo N (2008) Development of methodology for conducting clinical trials in frontotemporal lobar degeneration. Brain 131, 29572968.

[23] R Core Team (2015) R: A language and environment for statistical computing.Vienna, Austria, http://www.Rproject.org/

[24] Horn JL (1965) A rationale and test for the number of factors in factor analysis. Psychometrika 30, 179-185.

[25] Hayton JC, Allen DG, Scarpello V (2004) Factor retention decisions in exploratory factor analysis: A tutorial on parallel analysis. Organ Res Methods 7, 191-205.

[26] McKhann G, Knopman DS, Chertkow H, Hymann B, Jack CR, Kawas C, Klunk W, Koroshetz W, Manly J, Mayeux R, Mohs R, Morris J, Rossor M, Scheltens P, Carrillo M, Weintrub S, Phelphs C (2011) The diagnosis of dementia due to Alzheimer's disease: Recommendations from the National Institute on Aging- Alzheimer's Association workgroups on diagnostic guidelines for Alzheimer's disease. Alzheimers Dement 7, 263-269.

[27] Rascovsky K, Hodges JR, Knopman D, Mendez MF, Kramer JH, Neuhaus J, Van Swieten JC, Seelaar H, Dopper EG, Onyike CU, Hillis AE, Josephs KA, Boeve BF, Kertesz A, Seeley WW, Rankin KP, Johnson JK, Gorno-Tempini ML, Rosen H, Prioleau-Latham CE, Lee A, Kipps CM, Lillo P, Piguet O, Rohrer JD, Rossor MN, Warren JD, Fox NC, Galasko D, Salmon DP, Black SE, Mesulam M, Weintraub S, Dickerson BC, Diehl-Schmid J, Pasquier F, Deramecourt V, Lebert F, Pijnenburg Y, Chow TW, Manes F, Grafman 
J, Cappa SF, Freedman M, Grossman M, Miller BL (2011) Sensitivity of revised diagnostic criteria for the behavioural variant of frontotemporal dementia. Brain 134, 2456-2477.

[28] Morris JC (1997) Clinical dementia rating: A reliable and valid diagnostic and staging measure for dementia of the Alzheimer type. Int Psychogeriatr 9(Suppl 1), 173-176; discussion 177-178.

[29] Defrin R, Amanzio M, de Tommaso M, Dimova V, Filipovic S, Finn DP, Gimenez-Llort L, Invitto S, Jensen-Dahm C, Lautenbacher S, Oosterman JM, Petrini L, Pick CG, Pickering G, Vase L, Kunz M (2015) Experimental pain processing in individuals with cognitive impairment: Current state of the science. Pain 156, 1396-1408.

[30] Neary D, Snowden JS, Gustafson L, Passant U, Stuss D, Black S, Freedman M, Kertesz A, Robert PH, Albert M, Boone K, Miller BL, Cummings J, Benson DF (1998) Frontotemporal lobar degeneration: A consensus on clinical diagnostic criteria. Neurology 51, 1546-1554.

[31] Lavenu I, Pasquier F, Lebert F, Petit H, Van der Linden M (1999) Perception of emotion in frontotemporal dementia and Alzheimer disease. Alzheimer Dis Assoc Disord 13, 96101.

[32] Rosen HJ, Pace-Savitsky K, Perry RJ, Kramer JH, Miller BL, Levenson RW (2004) Recognition of emotion in the frontal and temporal variants of frontotemporal dementia. Dement Geriatr Cogn Disord 17, 277-281.

[33] Keane J, Calder AJ, Hodges JR, Young AW (2002) Face and emotion processing in frontal variant frontotemporal dementia. Neuropsychologia 40, 655-665.

[34] Davis MH (1983) Measuring individual differences in empathy: Evidence for a multidimensional approach. J Pers Soc Psychol 44, 113-126.

[35] Miller BL, Hou CE (2004) Portraits of artists: Emergence of visual creativity in dementia. Arch Neurol 61, 842-844.

[36] Ghacibeh GA, Heilman KM (2013) Creative innovation with temporal lobe epilepsy and lobectomy. J Neurol Sci 324, 45-48.

[37] Hagen EH, Bryant GA (2003) Music and dance as a coalition signaling system. Hum Nat 14, 21-51.

[38] Pearce E, Launay J, Dunbar RI (2015) The ice-breaker effect: Singing mediates fast social bonding. $R$ Soc Open Sci 2, 1-9.

[39] Moniz-Cook E, Agar S, Gibson G, Win T, Wang M (1998) A preliminary study of the effects of early intervention with people with dementia and their families in a memory clinic. Aging Ment Health 2, 199-212. 